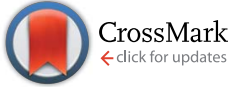

Cite this: RSC Adv., 2017, 7, 10973

Received 11th November 2016

Accepted 6th February 2017

DOI: $10.1039 / c 6 r a 26675 d$

rsc.li/rsc-advances

\section{The severe impact of in vivo-like microfluidic flow and the influence of gemini surfactants on amyloid aggregation of hen egg white lysozyme $\uparrow$}

\begin{abstract}
W. Gospodarczyk ${ }^{a}$ and M. Kozak ${ }^{\star a b}$
The formation of amyloid plaques is being intensively studied, as this process underlies severe human diseases, including Alzheimer's disease, and the exact mechanism of this specific aggregation has not been resolved yet. The investigation of its formation is accompanied by the search for substances inhibiting this aggregation process. Here we studied the process of hen egg white lysozyme (HEWL) amyloid aggregation process in bulk solution within a time scale of about a week. We also examined the influence of four dicationic (gemini) surfactants, including 3,3'-[1,6-(2,5-dioxahexane)]bis-(1dodecylimidazolium) dichloride (oxyC2), as well as selected reference sulfobetaines, on the aggregation process and found that at certain concentrations gemini surfactants remarkably inhibited amyloidogenesis. A microfluidic system was designed to mimic the in vivo-like conditions of flow in order to assess the influence of flow on the amyloid formation process. The flow was found to induce severe amyloid formation in just a few hours of exposition to the conditions which without flow would induce amyloidogenesis within a few days. The results shed light on the mechanisms responsible for the amyloid aggregation process and make an important contribution to understanding of the process.
\end{abstract}

\section{Introduction}

Amyloid aggregation (AA) is a specific type of protein aggregation process responsible for a number of serious human conformational diseases (proteinopathies), including Alzheimer's disease, Parkinson's disease and others, such as liver cirrhosis or type II diabetes. ${ }^{\mathbf{1 - 4}}$ Although scientists have made much effort to understand processes underlying this fatal aggregation in recent decades, the exact mechanism of AA still remains unclear. ${ }^{5}$ This knowledge would be of crucial importance in the quest for a drug against AA. ${ }^{6}$ As different amyloid proteins misfold by following a similar pathway, including pronounced $\beta$-sheet content rise, there is a hope of finding one drug for a few diseases at once. ${ }^{7,8}$ As the second main strategy in tackling amyloid aggregation challenge, besides attempting to disclose the molecular mechanism of amyloidogenesis, many researchers have also tested inhibitory effect of various substances on AA, with the ultimate aim of finding drug among them. ${ }^{9-11}$

Amid other approaches, there has been a growing interest in the use of microfluidics, which is a branch of science and

${ }^{a}$ Department of Macromolecular Physics, Faculty of Physics, Adam Mickiewicz University, Poznań, Poland.E-mail: mkozak@amu.edu.pl

${ }^{b}$ Joint Laboratory for SAXS Studies, Faculty of Physics, Adam Mickiewicz University, Umultowska 85, 61-614 Poznań, Poland

$\dagger$ Electronic supplementary information (ESI) available. See DOI: $10.1039 /$ c6ra26675d technology that deals with miniaturized devices called microchips, comprising channels of small cross sections, in AA research. ${ }^{12-14} \mathrm{~A}$ number of applications of microfluidics has been reported, which helped to overcome limitations of traditional, 'bulk' methods or enhance their potential. The microfluidics offers the ability to detect single nucleation sites of amyloidogenesis and trace their propagation in time, ${ }^{15}$ determine the influence of the flow rate of a sample flowing in a chip on the morphology of amyloid aggregates ${ }^{\mathbf{1 6}}$ and permits rapid profiling of specific proteins in bodily fluids. ${ }^{5}$ Several research groups have employed microchips which are designed to mimic in vivo-like flow conditions to model the effect of confinement and flow on the phenomena examined. ${ }^{\mathbf{1 7 - 2 0}}$ However, very few groups have tested long circulation inside a chip $^{21}$ and in order to find out how this process, entailing the occurrence of shear forces, can influence AA.

In this study we attempted to address both of the two needs: decipher AA molecular mechanism and screen for AA inhibitors. We therefore applied a microfluidic chip to test the influence of continued circulation of protein solutions on the AA process. We selected a well-studied model amyloid-prone protein (hen egg white lysozyme, HEWL) as the object of study. HEWL is an enzyme taking part in bacteria lysis which is homological to human lysozyme. It is a rather small, comprising 129 amino acid residues protein, whose secondary structure is dominated by $\alpha$-helices..$^{22,23}$ Secondly, we also tested the inhibitory effect of four dicationic (gemini) surfactants: $3,3^{\prime}$ [1,6-(2,5-dioxahexane)]bis-(1-dodecylimidazolium) dichloride 
Gemini surfactants:<smiles>CCCn1cc[n+](COCCOCCn2cc[n+](CC)c2)c1</smiles>

oxyCn

$n=2,4,8,12$

Sulfobetaines:

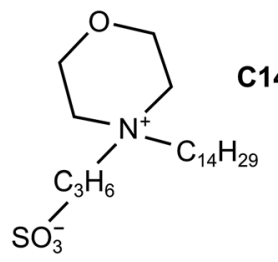

C14S3

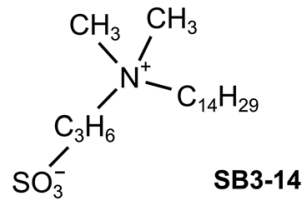

Fig. 1 Chemical structure of surfactants used in this study.

(oxyC2), 3,3'-[1,8-(2,7-dioxaoctane)]bis-(1-dodecylimidazolium) dichloride (oxyC4), 3,3'-[1,12-(2,11-dioxadodecane)]bis-(1-dodecylimidazolium) dichloride (oxyC8) and 3,3'-[1,16-(2,15-dioxahexadecane)]bis-(1-dodecylimidazolium) dichloride (oxyC12), ${ }^{24}$ and selected reference sulfobetaines: 3-( $N$-tetradecylmorpholine)-1-propanesulfonate (C14S3), 3-(,$N$-dimethylmyristylammonio)propanesulfonate (SB3-14) on AA of lysozyme (see chemical structures in Fig. 1). The surfactants selected for the study have different structures (dimeric or monomeric) and properties (cationic and zwitterionic). Gemini surfactants consist of two polar heads with two hydrophobic chains attached, and a linker group (also known as a spacer group) located between the heads. In comparison to their conventional (monomeric) surfactant counterparts, they exhibit unusual properties, such as higher surface activity, better solubility or lower critical micellization concentration and are a promising group of surfactants in the context of amyloidogenesis research. ${ }^{25-27}$ Sulfobetaines, on the other hand, were chosen as zwitterionic surfactants, having two electric charges of opposite signs, and having one hydrophobic chain. Amyloidogenesis inhibitors has been intensively sought among similar compounds. In addition, sulfobetaines are considered non-toxic, what is an additional asset for a prospective amyloid inhibitor. The microfluidic system we used is not a widely used option in microfluidic research, where the microchip usually serves as a mixing, droplet-forming, single-flow system, rarely as a mainly circulatory system. ${ }^{28-31}$ The principal goal of this work was to come closer to understanding the in vivo amyloidogenesis process by studying how the process of flow can be responsible for formation of amyloids.

\section{Experimental}

\subsection{Materials}

Hen egg white lysozyme was purchased from Carl Roth. All four gemini surfactants used in the study were synthesized in a way described earlier ${ }^{32}$ and were a generous gift from Dr Andrzej Skrzypczak (Poznań University of Technology, Poland). Thioflavin T, phosphate salts and phosphoric acid used for buffer preparation and guanidinium chloride were purchased from Sigma Aldrich. Sulfobetaine derivatives ${ }^{33-35}$ were a generous gift from Prof. Ryszard Zieliński (Poznań University of Economics) or purchased in Sigma-Aldrich (SB3-14).

\subsection{Amyloid aggregation of lysozyme}

Lysozyme amyloids were obtained as follows. ${ }^{36}$ A $10 \mathrm{mg} \mathrm{mL} \mathrm{m}^{-1}$ solution of HEWL in $50 \mathrm{mM}$ phosphate buffer at $\mathrm{pH}=2.0$ (obtained by mixing equally concentrated solutions of phosphoric acid and dihydrogen phosphate in a ratio of $1: 1.413$ ) was incubated at temperature of $60{ }^{\circ} \mathrm{C}$ for a time of 10 days or more. Amyloid aggregates were formed in these conditions gradually throughout the period of a week, with a changeable delay of the onset of the process. Aliquots were taken away from these samples at certain time intervals for carrying out ex situ measurements with use of diverse techniques. Therefore, if not stated otherwise, the default protein concentration in each experiment was unchanged, equal approximately $10 \mathrm{mg} \mathrm{mL} \mathrm{m}^{-1}$.

\subsection{Thioflavin $\mathbf{T}$ assay}

Thioflavin $\mathrm{T}$ (ThT) assay was performed according to a standard protocol. The portion of $8 \mathrm{mg}$ of powdered ThT was dissolved in $10 \mathrm{~mL}$ of $10 \mathrm{mM}$ phosphate buffer, $\mathrm{pH}=7.4$ (obtained by mixing equally concentrated solutions of dihydrogen phosphate and hydrogen phosphate in a ratio of $1: 0.288$ ), and kept in darkness afterwards for a few days as a stock solution. To obtain working solution on the day of thioflavin $\mathrm{T}$ fluorescence experiment, a small amount of stock solution was diluted 200 times in $10 \mathrm{mM}$ phosphate buffer. Then small aliquots of samples (always containing protein in concentration of about $10 \mathrm{mg} \mathrm{mL}{ }^{-1}$ ) were added to the working solution so that the final concentration of ThT was $12.5 \mu \mathrm{M}$ and the concentration of diluted HEWL was of about $50 \mu \mathrm{g} \mathrm{mL} \mathrm{mL}^{-1}(3.6 \mu \mathrm{M})$.

\subsection{Circular dichroism (CD)}

Circular dichroism measurements were carried out with the use of J-815 spectropolarimeter (Jasco, Japan). CD graphs were collected over the spectral range of 190-260 $\mathrm{nm}$ and the path length was equal to $0.01 \mathrm{~mm}$. Other parameters were: scanning speed: $50 \mathrm{~nm} \mathrm{~s}^{-1}$, spectral width: $0.5 \mathrm{~nm}$, number of accumulations: 5 , nitrogen flow rate: $10 \mathrm{~L} \mathrm{~min}^{-1}$. The data were deconvoluted at Dichroweb server ${ }^{37}$ to determine the secondary structure of protein. CDSSTR program module ${ }^{38}$ and SP175 database $^{39}$ were used for this aim. To perform CD measurements, the samples were not diluted and the HEWL concentration was of about $10 \mathrm{mg} \mathrm{mL} \mathrm{m}^{-1}$. However, as the protein concentration was subject to vary in particular experiments, the concentration was monitored ex situ using a NanoDrop ND1000 microspectrophotometer. Guanidinium chloride was added to the samples to improve the measurement accuracy, in case that large protein aggregates could interfere with spectroscopic measurements. However, the guanidinium chloride addition did not disturb CD (nor any other) measurements. In practice, an aliquot of each sample subject to protein concentration measurement was taken away from the sample and was subsequently mixed with $8 \mathrm{M}$ guanidinium chloride solution in a ratio of $1: 1$ for concentration measurement only. 


\subsection{Spectrofluorimetry}

Spectrofluorimetry was used to determine fluorescence signal from thioflavin $\mathrm{T}$ that is bound specifically to amyloid aggregates, which is well known in the field as an indicator of formation of protein amyloids. Relevant data were collected with FP-6300 spectrofluorimeter (Jasco, Japan) for the following measurement parameters: excitation wavelength $450 \mathrm{~nm}$, bandwidth $5 \mathrm{~nm}$; the emission wavelength range $470-560 \mathrm{~nm}$, bandwidth $10 \mathrm{~nm}$. The measurements were carried out in cuvettes of the path length of $10 \mathrm{~mm}$, at the scanning speed of $100 \mathrm{~nm} \mathrm{~min}{ }^{-1}$. The final data set was obtained after 3 accumulations.

\subsection{Small angle X-ray scattering (SAXS)}

Small angle X-ray measurements were performed at P12 beam line of Deutsches Elektronen-Synchrotron, PETRA III storage ring, (Hamburg, Germany). ${ }^{\mathbf{4 0 - 4 2}}$ A hybrid photon counting Pilatus $2 \mathrm{M}$ pixel detector $\left(253 \times 288 \mathrm{~mm}^{2}\right)$ was used, with sampleto-detector distance equal to $3500 \mathrm{~mm}$. A calibration of detector $s$-axis was made, with the use of diffraction patterns of silver behenate. ${ }^{43}$ The scattering vector $(s)$ here stands for $4 \pi \sin \theta / \lambda$, where $2 \theta$ is the scattering angle, and $\lambda$ is the synchrotron radiation wavelength, which was equal $0.15 \mathrm{~nm}$. SAXS data were collected in the $s$ range of $0.08 \mathrm{~nm}^{-1}$ to $4.5 \mathrm{~nm}^{-1}$. Twenty frames, with $0.1 \mathrm{~s}$ exposure time, recorded for each sample, were subsequently integrated and averaged. Measurements for all samples were made at temperature of $10{ }^{\circ} \mathrm{C}$, using a $10 \mu \mathrm{L}$ capillary cell with automated filling. SAXS data were processed with the use of PRIMUS program package. ${ }^{44}$ Xylose/glucose isomerase from Streptomyces rubiginosus solution, with a known concentration of $3 \mathrm{mg} \mathrm{mL}^{-1}$, was used as a reference. ${ }^{45}$ For all SAXS experiments, the HEWL concentration was unchanged and kept the same as in CD experiments.

To derive information about protein molecules conformation changes or growth in particles size due to aggregation onset, the radii of gyration were estimated from the experimental SAXS data. According to Guinier relation, the natural logarithm of intensity of the scattering curve is linear with $s^{2}$ (for small $s$ values), and the slope coefficient is proportional to $R_{\mathrm{g}}{ }^{2}$. Relevant $R_{\mathrm{g}}$ values were determined with the use of PRIMUS, with respect to necessity that $s R_{\mathrm{g}}$ product should stay between 0.1 and 1.5.

\subsection{Transmission electron microscopy (TEM)}

Transmission electron microscopy images were obtained with the use of a JEOL JEM-1200EX II Electron Microscope, for $80 \mathrm{kV}$ high voltage. For each experiment, HEWL samples were diluted 30 times and then placed on carbon-coated copper grids for 2 minutes. Then, the sample was removed and staining solution was placed on grids instead, again for 2 minutes time. The staining solution was uranyl formate, $2 \%(\mathrm{w} / \mathrm{v})$.

\subsection{Microfluidic system}

The microfluidic system used in the study comprised a system of pressure controllers, pumps and a microchip. The pressure controller, denoted as OB1 (OB1PC), was purchased from
Elvesys Microfluidic Innovation Centre, Paris, France. An external pressure source (bottle of nitrogen, purity 99.99\%) was connected to OB1PC. The controller exerted desired pressure from the range of $4 \mathrm{mbar}$ to $8000 \mathrm{mbar}$ on the sample placed in an Eppendorf tube. The sample, pushed by this pressure, was directed into tubing and flowed towards the microchip. After having travelled through the chip, the sample was collected by another Eppendorf tube. The pressure from OB1PC can be delivered to either of the Eppendorf tubes, and the flow can therefore be bidirectional. In this way a constant bi-directional circulation of sample through the chip was realized.

The glass microfluidic chip was purchased from Dolomite Microfluidics, Canterbury, England. It comprises one serpentine-like 5 metre long channel of a $100 \mu \mathrm{m} \times 220 \mu \mathrm{m}$ cross-section in a shape of a rectangular with two curved corners. Its internal volume is equal to $90 \mu \mathrm{L}$. The microfluidic chip together with Eppendorf tubes and tubing was mounted on a heating stage to keep the system at desired temperature in a stable way. The average flow rate could easily be calculated by measuring the time of flow of the sample from a particular point of the system to another one, when the volume of the sample flowing inside the system was known.

All above mentioned elements of the system are shown in Fig. 2. During the experiment, the average flow rate was kept approximately at $50 \mu \mathrm{L} \mathrm{min}{ }^{-1}$ or $100 \mu \mathrm{L} \mathrm{min}^{-1}$, or $20 \mu \mathrm{L} \mathrm{min}^{-1}$. At the beginning of the experiments, a total volume of $150 \mu \mathrm{L}$ of the sample was put into the system. However, in some series of experiments the initial sample volume could be different, e.g. $200 \mu \mathrm{L}$.

Under physiological conditions the occurring shear rates ${ }^{46}$ are in the range of $100-8000 \mathrm{~s}^{-1}$. Sorteberg et al. ${ }^{47}$ report that some brain arteries can carry blood at velocities as high as $50 \mathrm{~cm} \mathrm{~s}^{-1}$. The vessels diameters can vary from tenths of micrometer to a few millimetres. ${ }^{48,49}$ These examples prove that flow inside human organism is remarkably diverse. This fact is clearly reflected in a very broad choice of parameters used in microfluidic experiments. For example, researchers aiming at mimicking the in vivo-like conditions in their studies chose flow rates ranging from $0.1 \mu \mathrm{L} \mathrm{min}{ }^{-1}$ to $3300 \mu \mathrm{L} \mathrm{min}^{-1}$, with average velocities from $0.1 \mathrm{~mm} \mathrm{~s}^{-1}$ to $100 \mathrm{~mm} \mathrm{~s}^{-1} \cdot{ }^{16,18,20,21,50,51}$ The parameters we used in our experiments are consistent with parameters of physiological flow and the flow regime we examined is therefore indeed physiologically relevant.

\section{Results and discussion}

\subsection{Amyloid aggregation of lysozyme samples in bulk conditions}

The amyloid aggregation process of HEWL was examined by circular dichroism, thioflavin T fluorescence, small angle X-ray scattering and transmission electron microscopy. Lysozyme at low pH was incubated as described in the Experimental section. The CD curves revealing the HEWL secondary structure after different times of incubation are shown in Fig. 3a. The corresponding ThT fluorescence signal values at $\lambda=490 \mathrm{~nm}$ derived from fluorescence spectra taken for these samples are shown in Fig. 3a inset. The CD spectra were deconvoluted to determine 

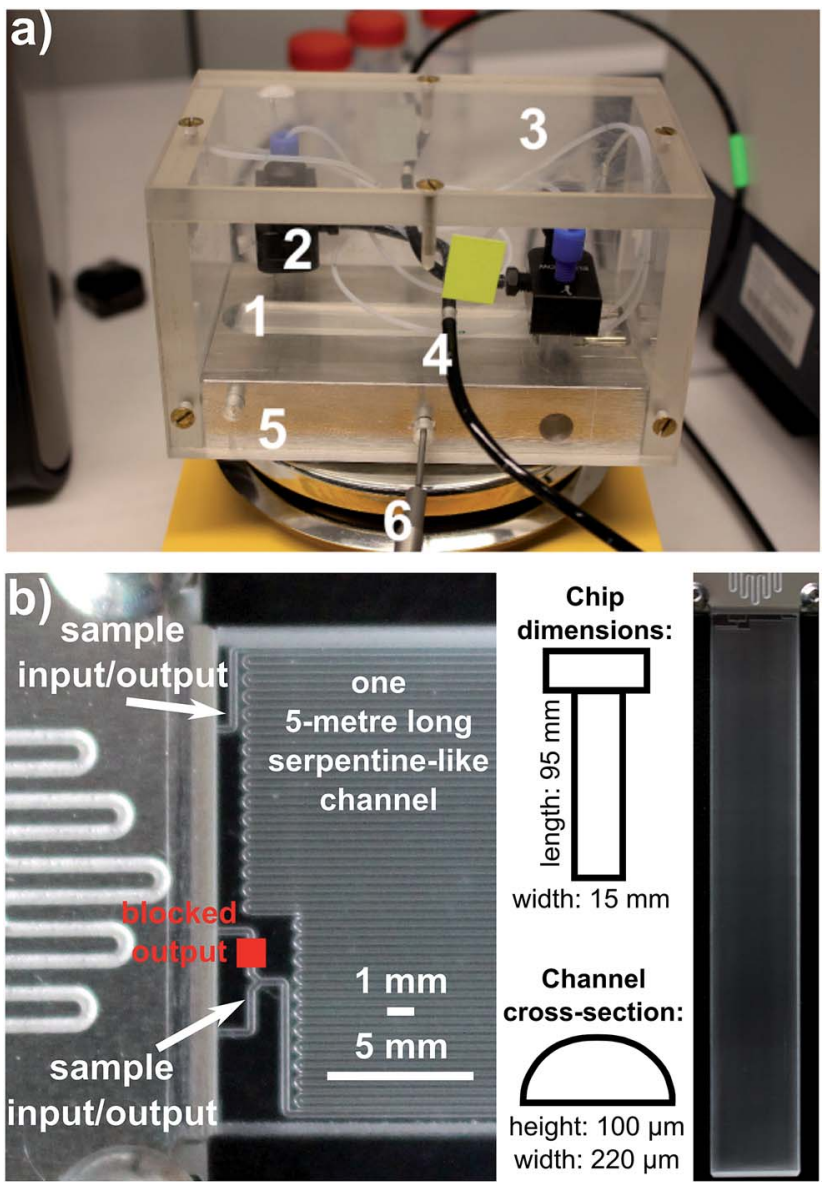

\section{c)}

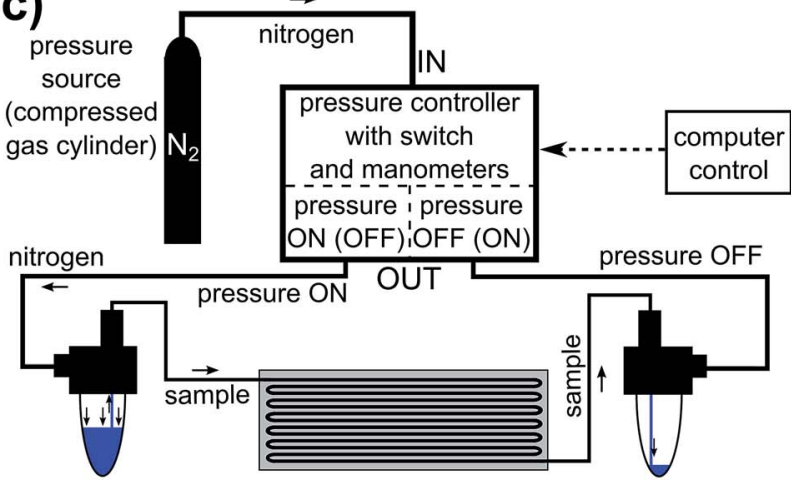

Fig. 2 Picture of experimental microfluidic system showing its front view (a). The following elements of the setup are shown by numbers: 1 - microfluidic chip; 2 - Eppendorf tube reservoirs; 3 - tubing connecting Eppendorf tubes with chip; 4 - tubing supplying Eppendorf tubes with pressure; 5 - heating block; 6 - temperature control. A detailed view of microchip, with chip dimensions (b). Schematic view of the experimental setup (c). The moment when sample is flowing from the left Eppendorf tube towards the right tube is caught in the scheme; the direction of flow is reversed when the pressure controller cuts off pressure in the left channel and applies pressure to the right channel.

HEWL secondary structure ( $\alpha$-helix and $\beta$-sheet content). The results of this deconvolution are given in Table 1 ('for 0 mM' column). The $\alpha$-helix content is gradually decreased in favor of $\beta$ sheet content; then, after 10 days of incubation, the content of $\alpha$ helix is partially retrieved. The increase in $\beta$-sheets content is
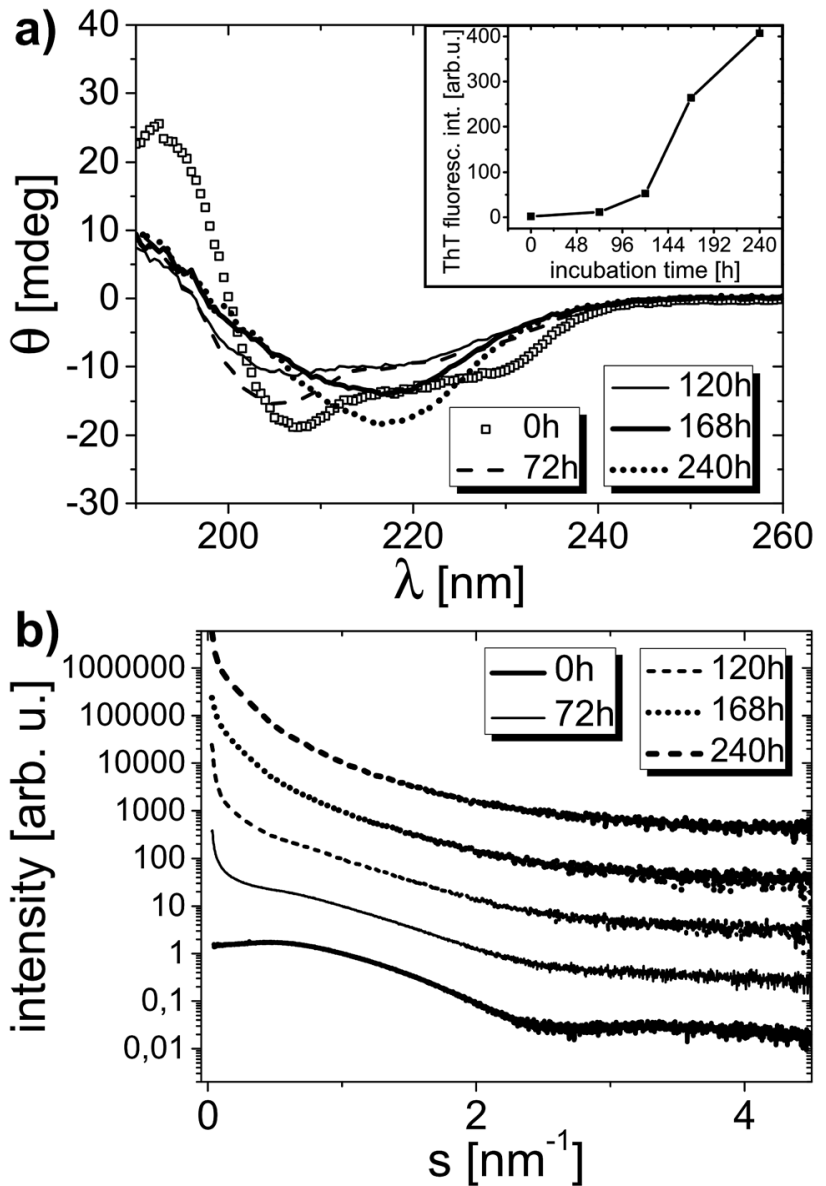

c)

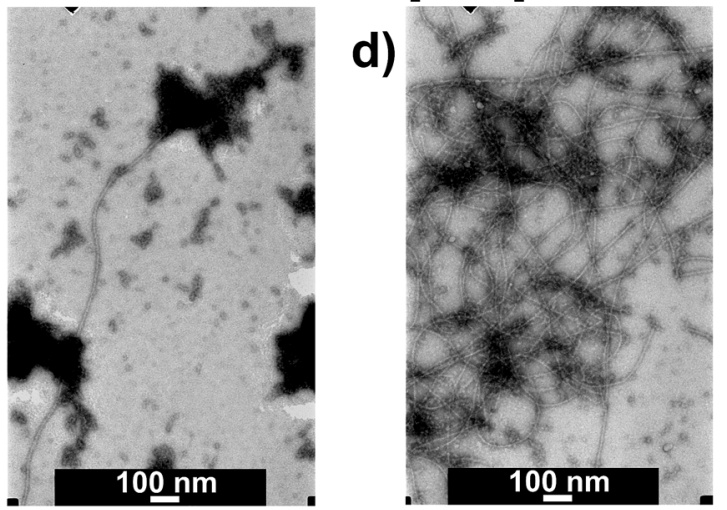

Fig. 3 Circular dichroism spectra collected for bulk solution of lysozyme without surfactant after given time of incubation (a). Thioflavin $T$ fluorescence signal at $\lambda=490 \mathrm{~nm}$ in dependence on time of incubation, measured for the same sample (inset). Small angle $X$-ray scattering curves measured for the same sample under study (b). Curves were shifted vertically for clarity. TEM images of a sample containing lysozyme without surfactant after: 5 days of incubation (having $5 \mathrm{arb}$. $u$. of ThT fluorescence signal at $\lambda=490 \mathrm{~nm}$ ) (c) and 7 days of incubation (13 arb. u. at $\lambda=490 \mathrm{~nm}$ ) (d).

typical of amyloid formation. ThT fluorescence experiments confirm this observation as the rise in thioflavin $\mathrm{T}$ fluorescence signal is indicative of amyloidic aggregates presence in the solution studied. ${ }^{52}$ In accordance with ThT fluorescence results the deposits formation in the solution is apparently started from the third day on. 
Table 1 The secondary structure of HEWL calculated for HEWL/oxyC2 samples for given surfactant concentrations and time of sample incubation. The data were obtained as a result of deconvolution of $C D$ curves shown in Fig. 3a and S1a-c. SAXS data gathered for these samples are given in Fig. 3b and S2 (scattering curves) and summarized in Table 2 (radii of gyration)

\begin{tabular}{|c|c|c|c|c|}
\hline \multirow{3}{*}{$\begin{array}{l}\text { Time of incubation } \\
{[\mathrm{h}]}\end{array}$} & \multicolumn{4}{|c|}{ oxyC2 concentration } \\
\hline & \multicolumn{4}{|c|}{$\alpha$-Helix $/ \beta$-sheet content $[\%]$} \\
\hline & $0 \mathrm{mM}$ & $1 \mathrm{mM}$ & $5 \mathrm{mM}$ & $20 \mathrm{mM}$ \\
\hline 0 & $56 / 6$ & $57 / 5$ & $57 / 6$ & $55 / 5$ \\
\hline 72 & $35 / 12$ & $38 / 11$ & $39 / 10$ & $40 / 12$ \\
\hline 120 & $22 / 24$ & $30 / 17$ & $39 / 11$ & $41 / 11$ \\
\hline 168 & $22 / 31$ & $35 / 18$ & $40 / 13$ & $39 / 12$ \\
\hline 240 & $38 / 25$ & $37 / 17$ & $39 / 13$ & $39 / 12$ \\
\hline
\end{tabular}

SAXS data collected for the same samples are plotted in Fig. $3 \mathrm{~b}$, and the corresponding $R_{\mathrm{g}}$ values derived from these curves are presented in Table 2 ('for 0 mM' column). According to the determined $R_{\mathrm{g}}$ values, the protein radius of gyration rises from $1.5 \mathrm{~nm}$ at the beginning to $8.1 \mathrm{~nm}$ at the end of incubation process. With increasing incubation time, the slope of the scattering curve increases especially in the range of small scattering vector. This is the result of the presence of protein particles (or aggregates) of growing radius of gyration in the solution. Starting from the third day of incubation, the solution becomes polydisperse, as there are two or more values of radius of gyration that could be determined within the accepted $s R_{\mathrm{g}}$ limit. The onset of aggregation process is therefore easy to identify from SAXS dependencies. Because the solutions become polydisperse, the determination of the exact radius of gyration is hampered and the values of radii of gyration determined from particular SAXS curves and presented in Table 2, do not necessarily mean that the sample contains only particles of the gyration radii from the range indicated. These values imply that protein pre-aggregates and aggregates are already there in the solution, meaning that AA has already been triggered in the lysozyme solution. Indeed, one should not conclude, for instance, that after 10 days of incubation there are only particles with well-defined $R_{\mathrm{g}}$ values from the 8.1-11.2 $\mathrm{nm}$ range present in the solution.

Table 2 Radii of gyration calculated for HEWL/oxyC2 samples for given surfactant concentrations and time of sample incubation. The data were derived from SAXS curves shown in Fig. 3b and S2

oxyC2 concentration

\begin{tabular}{|c|c|c|c|c|}
\hline \multirow{3}{*}{$\begin{array}{l}\text { Time of incubation } \\
{[\mathrm{h}]}\end{array}$} & \multicolumn{4}{|c|}{ oxyC2 concentration } \\
\hline & \multicolumn{4}{|l|}{$R_{\mathrm{g}}[\mathrm{nm}]$} \\
\hline & $0 \mathrm{mM}$ & $1 \mathrm{mM}$ & $5 \mathrm{mM}$ & $20 \mathrm{mM}$ \\
\hline & 1.5 & 1.5 & 1.6 & 1.5 \\
\hline 72 & $1.8-8.0$ & 1.5 & 1.9 & 1.8 \\
\hline 120 & $2.3-8.2$ & $2.2-9.7$ & $2.1-9.7$ & 1.8 \\
\hline & $8.0-9.3$ & 7.6-9.9 & $6.7-10.0$ & $1.9-2.1$ \\
\hline 24 & $8.1-11.2$ & $8.0-10.9$ & $7.8-9.1$ & $2.2-6.0$ \\
\hline
\end{tabular}

Also TEM micrographs confirm the amyloid aggregation process of lysozyme. Fig. 3c and d display two TEM images of one sample containing HEWL without surfactant on the fifth and the seventh day of incubation process, respectively. There are long and plentiful amyloid fibrils visible in the sample. A growing fraction of fibrils in the sample can be observed with rising incubation time.

According to additional measurements of ThT fluorescence signals obtained for different lysozyme-alone samples, it became evident that the onset time, as well as the extent of AA are sample dependent (Table $\mathrm{S} 1 \dagger$ ). As the particular parameters of amyloid pathway could vary from series to series, or even between samples from one series, it seems that this variation is an inherent feature of the phenomena examined, as the experimental conditions and procedures of samples preparation were identical in each case.

To summarize lysozyme-alone system properties, lysozyme sample in bulk conditions under incubation clearly undergoes amyloid aggregation. The amyloid growth takes days to develop, which is in good agreement with literature data..$^{53}$

\subsection{Amyloid aggregation of lysozyme-surfactant oxyC2 samples in bulk conditions}

Furthermore, we examined the possible inhibitory influence of dicationic surfactants (bis-imidazolium derivatives) and zwitterionic surfactants (sulfobetaine derivatives) studied on the amyloidogenic lysozyme aggregation process. Three different solutions, containing varying amounts of oxyC2 surfactant, were studied in the same way as the above-described lysozymealone samples. The circular dichroism results, including CD curves and calculated secondary structure content, are shown in Fig. S1 $\uparrow$ and Table 1, respectively. The corresponding ThT fluorescence curves were also measured (Fig. S1d†). It can be seen that for all four samples, including three samples with different surfactant concentrations and the lysozyme-alone sample, there is a remarked drop in the $\alpha$-helix content after three days of incubation. This comes, however, without a significant rise in ThT fluorescence signal intensity after this time, as the emission signal intensities at $490 \mathrm{~nm}$ after three days of incubation for the four samples made $2.8 \%$ (for $0 \mathrm{mM}$ of oxyC2), $3.1 \%$ (for $1 \mathrm{mM}$ ), $0.5 \%$ (for $5 \mathrm{mM}$ ) and $0.1 \%$ (for $20 \mathrm{mM}$ ) of the signal intensity for the sample containing lysozyme without surfactant after 10 days of incubation (shown in Fig. 3a inset). It must mean that in the first phase of amyloid aggregation process, HEWL is at first subjected to a partial, however pronounced, change in secondary structure prior to strong amyloidogenesis (which does not rule out the possible occurrence of a small number of fibrillar or proto-fibrillar aggregates in solution that exhibits slightly, but distinctly elevated ThT fluorescence level, what was the case in a sample shown in Fig. 3c). Amyloid aggregation process must therefore result from significant changes in the secondary structure $(\alpha$-helix drop and $\beta$-sheet content rise), which was anticipated as a well-known circumstance. This drop takes place regardless of surfactant addition, but is slightly more gentle for lysozymesurfactant samples. In the lysozyme sample without surfactant 
the drop is not finished on the third day of incubation and is extended to a further strong decrease in the $\alpha$-helix content (down to $22 \%$ ), before there are again more $\alpha$-helices on the tenth day of measurement. Such secondary structure dependence is also true for lysozyme/oxyC2 (1 mM) sample, but the abovementioned further drop is much smaller and is at an end at shorter time (on the seventh day). For higher surfactant concentrations, on the other hand, the secondary structure after primary drop is preserved. After ten days of experiment, all samples reveal similar $\alpha$-helix content, but different $\beta$-sheet content: the percentage of $\beta$-sheets is distinctly smaller for HEWL/oxyC2 (5 mM) and HEWL/oxyC2 (20 mM) samples than for HEWL/oxyC2 (1 mM) sample, whereas the greatest content of $\beta$-sheet structure occurs in the HEWL-alone sample. It can therefore be concluded that oxyC2 surfactant addition has a notable impact on the HEWL secondary structure, with a role of sustaining $\alpha$-helix and $\beta$-sheet contents.

The dependence of thioflavin $\mathrm{T}$ fluorescence emission signal intensities at $490 \mathrm{~nm}$ for HEWL/oxyC2 (1 mM) sample measured as a function of the day of incubation (Fig. S1d $\dagger$ ) is not much different from the same curve measured for HEWLalone solution, but AA is eventually slightly diminished (to $74 \%$ of the value of the signal for HEWL sample without surfactant after 10 days). Addition of higher amounts of gemini surfactant ( $5 \mathrm{mM}$ and $20 \mathrm{mM}$ ), however, leads to a serious cut of the ThT fluorescence emission intensity (to $32 \%$ for $5 \mathrm{mM}$ of oxyC2 and $0.0 \%$ for $20 \mathrm{mM}$ of oxyC2 after 10 days of incubation, with regard to the value measured for the lysozyme sample without surfactant after 10 days of incubation). For the sample of $20 \mathrm{mM}$ concentration of surfactant, the initial intensity of ThT fluorescence signal remains unchanged during incubation process. As thioflavin $\mathrm{T}$ fluorescence is characteristic of amyloid aggregates formation, one could conclude that oxyC2 $(20 \mathrm{mM})$ is a perfect AA inhibitor, especially that no deposits are seen in HEWL/oxyC2 (20 mM) solutions, even after 10 days of incubation. However, the SAXS curves and TEM images of these samples may suggest a slightly different option to occur.

In SAXS dependencies measured for lysozyme samples containing $1 \mathrm{mM}, 5 \mathrm{mM}$ and $20 \mathrm{mM}$ of oxyC2 surfactant (Fig. S2 $\dagger$ ), similarly to the curve shown in Fig. 3b, a steep slope of curves for small scattering vector values reveals formation of preaggregates, as discussed earlier for the sample without oxyC2. In HEWL-alone and HEWL-oxyC2 (1 mM) samples, a characteristic curve beginning is evident right from the third day of incubation. On the other hand, for HEWL-oxyC2 (5 mM) and HEWL-oxyC2 (20 $\mathrm{mM})$ samples, the aggregation onset is delayed, to the fifth and to the seventh day, respectively. This is an additional confirmation of the fact that oxyC2 surfactant has some inhibitory effect on the amyloidogenesis process in the samples studied. But, it also proves that oxyC2 in $20 \mathrm{mM}$ concentration does not totally prevent HEWL protein from amyloid aggregation, as could appear from the ThT fluorescence data. The above-discussed delayed aggregation onset as well as aggregation in HEWL-oxyC2 (20 mM) samples is also clearly revealed by the radii of gyration values derived from these SAXS curves, listed in Table 2.
The suppressed ThT fluorescence intensity of HEWL-oxyC2 (20 $\mathrm{mM}$ ), and also for other HEWL samples with dicationic surfactants (20 mM) samples, as will be discussed below, could have different possible explanations. We considered four potential scenarios: 1 - oxyC2 surfactant indeed inhibits AA, but may induce HEWL to form different, non-amyloid aggregates; 2 - oxyC2 binds to protein so tightly that it prevents thioflavin $\mathrm{T}$ from contact with protein; 3 - oxyC2 surfactant binds to thioflavin $\mathrm{T}$ disabling it to interact with HEWL; and 4 - oxyC2 absorbs efficiently in the spectral range of measurement. We easily dismissed the last scenario, as we measured that gemini surfactant in concentration $50 \mathrm{mM}, 4 \mathrm{~mm}$ path length, showed no greater absorption than buffer-alone solution in the spectral range from $300 \mathrm{~nm}$ to $700 \mathrm{~nm}$. We also checked that ThT fluorescence signal intensity only halved after adding surfactant to the solution of already formed amyloids. This is a result contradicting the third scenario. On the basis of our data gathered in all experiments made, we cannot exclude the explanation no. 2, whereas in the light of TEM results obtained for a greater number of different surfactants, discussed later on, we may claim that the first option definitely makes a great contribution to the observed ThT fluorescence suppression.

Fig. 4 presents TEM images of a different series of samples with the same composition. Fibril-like structures are observed in HEWL/oxyC2 (1 mM) sample after 7 days of incubation, but there are fewer of them in HEWL/oxyC2 (5 mM) sample, and even fewer in HEWL/oxyC2 (20 $\mathrm{mM})$ sample. In the latter sample, the fibrils are hard to find. After 10 days, in the sample containing $1 \mathrm{mM}$ of oxyC2 surfactant, there are many fibril-like aggregates, but they are less visible in the image as it seems they
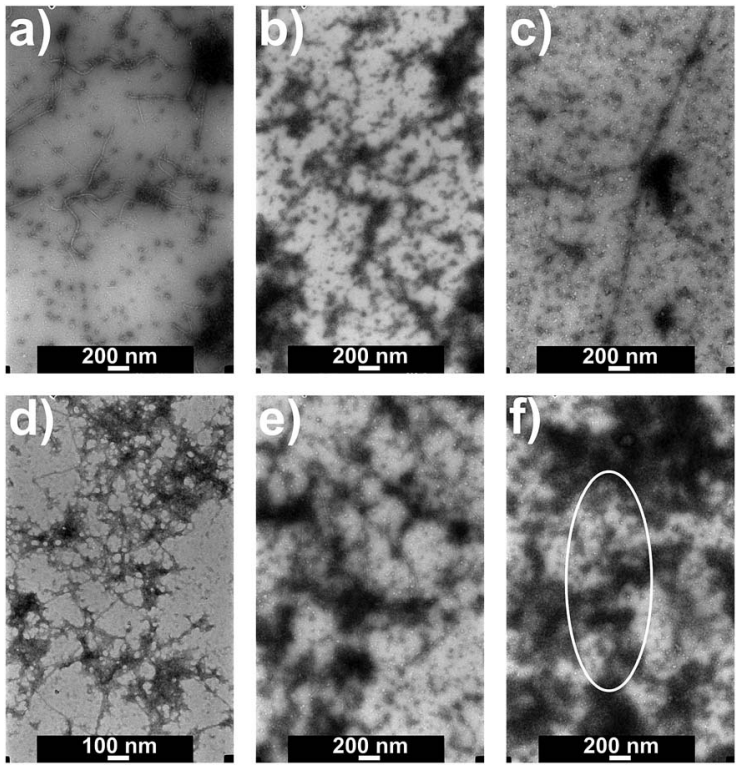

Fig. 4 TEM images of HEWL-OxyC2 surfactant samples, for different surfactant concentrations and times of incubation: $1 \mathrm{mM}, 7$ days (sample exhibiting $25 \mathrm{arb}$. $u$. of ThT fluorescence signal at $\lambda=490 \mathrm{~nm}$ ) (a); 5 mM, 7 days (3.6 arb. u.) (b); 20 mM, 7 days ( -0.6 arb. u.) (c); 1 mM, 10 days (42 arb. u.) (d); 5 mM, 10 days (4.0 arb. u.) (e); 20 mM, 10 days $(0.0$ arb. u.) (f). The white oval in (f) highlights a hardly visible, but distinct long lysozyme fibrillar aggregate found in the sample. 
could be coated with some material. There is a number of fibrillar aggregates in the sample with $5 \mathrm{mM}$ of oxyC2, but for the sample with $20 \mathrm{mM}$ of oxyC2 surfactant, there are very few of them, as the one rounded in Fig. 4f with a white oval. The addition of oxyC2 surfactant, especially in the highest tested surfactant concentration, indeed prevents the AA development.

To sum this part up, oxyC2 surfactant has a non-negligible impact on HEWL amyloid aggregation, by preserving protein secondary structure, delaying the aggregation onset and diminishing the extent of AA.

\subsection{Amyloid aggregation of lysozyme with other surfactants in bulk conditions}

Besides the thoroughly examined HEWL/oxyC2 system, also the influence of other surfactants on protein amyloidogenesis was studied. As the amyloid aggregation onset time as well as the extent of AA vary from one series of samples to another, to compare the impact of different surfactants on system properties, we prepared one series containing additions of all surfactants taken under study. For the different gemini surfactants: oxyC2, oxyC4, oxyC8 and oxyC12, we examined HEWLsurfactant samples with surfactants concentrations of $5 \mathrm{mM}$ and $20 \mathrm{mM}$ only. For both sulfobetaine derivatives, $50 \mathrm{mM}$ concentration was investigated. Also two reference lysozymealone samples were made.

ThT fluorescence results are shown in Table 3. In this series, the intensity of emission signal from lysozyme-alone samples reached about 55 arb. u. after 31 days of sample heating. This rise of fluorescence intensity is diminished for HEWL-gemini surfactant samples. However, as discussed above, this observation could not be a conclusive evidence of the lack of amyloid aggregates. Again, in samples containing oxyCn $(20 \mathrm{mM})$ surfactant, surfactant suppressed ThT fluorescence signal rise stronger than in oxyCn $(5 \mathrm{mM})$ samples. For oxyCn $(5 \mathrm{mM})$ samples, an apparent effect of the ThT signal saturation and the

Table 3 Thioflavin T fluorescence signal at $\lambda=490 \mathrm{~nm}$ for one series of HEWL samples without surfactant addition and with addition of different surfactants, with their concentration given in the table, as a function of day of incubation

\begin{tabular}{|c|c|c|c|c|c|}
\hline \multirow{3}{*}{$\begin{array}{l}\text { Surfactant } \\
\text { type }\end{array}$} & \multirow{3}{*}{$\begin{array}{l}\text { Surfactant } \\
\text { conc. }[\mathrm{mM}]\end{array}$} & \multicolumn{4}{|c|}{$\begin{array}{l}\text { Number of days of } \\
\text { incubation }\end{array}$} \\
\hline & & \multicolumn{4}{|c|}{$\begin{array}{l}\text { ThT fluorescence intensity } \\
\text { [arb. u.] }\end{array}$} \\
\hline & & 0 & 14 & 21 & 31 \\
\hline \multirow[t]{2}{*}{ No surfactant } & 0 & 2 & 5 & 15 & 55 \\
\hline & & 2 & 15 & 22 & 53 \\
\hline \multirow[t]{2}{*}{ oxyC2 } & 5 & 1 & 8 & 11 & 7 \\
\hline & 20 & -1 & 0 & 1 & 2 \\
\hline oxyC4 & 20 & -1 & 0 & 2 & 5 \\
\hline \multirow[t]{2}{*}{ oxyC8 } & 5 & 0 & 16 & 20 & 8 \\
\hline & 20 & 0 & 0 & 3 & 5 \\
\hline oxyC12 & 20 & 2 & -1 & 2 & 4 \\
\hline C14S3 & 50 & 2 & 8 & 44 & 33 \\
\hline SB3-14 & 50 & 1 & 4 & 15 & 48 \\
\hline
\end{tabular}

ThT signal drop at the end of the experiment. Sulfobetaines, on the other hand, show limited or no effect on AA inhibition. The ThT fluorescence intensity values are comparable to those obtained for HEWL-alone samples.

The secondary structure of proteins deconvoluted for this series is given in Table 4. We encountered some difficulties in obtaining fully conclusive exact protein concentration values, despite a few attempts made, which seem to be natural for these samples. The contents of $\alpha$-helical and $\beta$-sheet are therefore approximate, revealing, however, valuable information about trends in the system evolution and allowing a comparison between surfactants, regarding the effect each of them exerts on HEWL amyloidogenesis. One may expect the real secondary structure to be $\alpha$-helix richer and $\beta$-sheets poorer. Nonetheless, it can be clearly seen that the extent to which $\alpha$-helix content in HEWL-alone samples is gradually decreased is weakened for HEWL/oxyCn systems. For HEWL/oxyC4 (20 mM) sample, after initial drop in the content of $\alpha$-helical structure, the amount of $\alpha$-helices is constant. Similar dependence was observed for HEWL/oxyC2 (20 mM) sample, but for longer spacer gemini surfactants, oxyC8 and oxyC12, the reduction of $\alpha$-helical structure is greater than for gemini surfactants with shorter spacers. Additionally, at higher surfactant concentrations, oxyC2 and oxyC8 surfactants show greater inhibitory properties, but this effect is far more pronounced for the surfactant with the shorter spacer. Sulfobetaine derivatives, contrary to dicationic surfactants, modify the HEWL secondary structure at the very beginning of the experiment. Then, the HEWL secondary structure changes are smaller than in the case of gemini surfactants, and the final secondary structure is preserved to a similar extent as for oxyC2 $(20 \mathrm{mM})$ and oxyC4 $(20 \mathrm{mM})$ samples.

TEM images were taken of all the samples from this series at the final stage of incubation (Fig. S3†). There are lots of fibrillar aggregates visible in both 'no surfactant' samples, but one of the two, shown in Fig. S3b, $\uparrow$ has a smaller number of them. There is a great number of aggregates observed for SB3-14

Table 4 Secondary structure of lysozyme calculated for the series of HEWL samples without surfactant addition and with addition of different surfactants, at concentration given in the table, presented as a function of day of incubation

\begin{tabular}{|c|c|c|c|c|c|}
\hline \multirow{3}{*}{$\begin{array}{l}\text { Surfactant } \\
\text { type }\end{array}$} & \multirow{3}{*}{$\begin{array}{l}\text { Surfactant } \\
\text { concentration }[\mathrm{mM}]\end{array}$} & \multicolumn{4}{|c|}{$\begin{array}{l}\text { Number of days of } \\
\text { incubation }\end{array}$} \\
\hline & & \multicolumn{4}{|c|}{$\alpha$-Helix/ $\beta$-sheet content $[\%]$} \\
\hline & & 0 & 14 & 21 & 31 \\
\hline \multirow[t]{2}{*}{ No surfactant } & 0 & $52 / 7$ & $21 / 20$ & $18 / 23$ & $15 / 25$ \\
\hline & & $55 / 6$ & $18 / 23$ & $17 / 24$ & $8 / 32$ \\
\hline \multirow[t]{2}{*}{ oxyC2 } & 5 & $55 / 7$ & $32 / 15$ & $24 / 19$ & $19 / 23$ \\
\hline & 20 & $57 / 5$ & $37 / 13$ & $34 / 14$ & $31 / 16$ \\
\hline oxyC4 & 20 & $56 / 6$ & $36 / 12$ & $34 / 14$ & $33 / 14$ \\
\hline \multirow[t]{2}{*}{ oxyC8 } & 5 & $54 / 6$ & $38 / 12$ & $30 / 18$ & $19 / 23$ \\
\hline & 20 & $54 / 5$ & $35 / 14$ & $31 / 15$ & $22 / 21$ \\
\hline oxyC12 & 20 & $56 / 6$ & $34 / 13$ & $32 / 16$ & $22 / 23$ \\
\hline C14S3 & 50 & $44 / 9$ & $42 / 10$ & $34 / 15$ & $30 / 16$ \\
\hline SB3-14 & 50 & $39 / 12$ & $39 / 10$ & $35 / 13$ & $31 / 17$ \\
\hline
\end{tabular}


sulfobetaine, and distinctly fewer fibrils for C14S3, but due to grid foil crack only a small area of this sample was looked through. There were very few amyloid aggregates visible in all dicationic surfactants $20 \mathrm{mM}$ samples with a minor exception of oxyC12 $20 \mathrm{mM}$ sample, where markedly more aggregates could be seen, but still markedly fewer than in the samples without surfactant and with sulfobetaines. Amyloid aggregates were clearly more abundant in the $5 \mathrm{mM}$ samples as compared to the respective $20 \mathrm{mM}$ solutions. Fibrils in the samples were often seen as if they were coated with some substance, which could expectedly be surfactant micelles or other surfactant structures, or non-amyloid protein aggregates.

Finally, SAXS patterns were collected for different lysozymesurfactants systems. For oxyC4, oxyC8 and oxyC12 HEWL/ gemini surfactants samples the characteristic steep slope in scattering curves was observed, similar to that noted for HEWL/ oxyC2 samples (data not shown). It means that, similarly to oxyC2 in HEWL/oxyC2 system, the gemini surfactants studied in $5 \mathrm{mM}$ and $20 \mathrm{mM}$ concentrations delayed the aggregation onset, except for oxyC12 surfactant.

Because there were considerably smaller numbers of fibrillar aggregates found in HEWL with gemini surfactant $(20 \mathrm{mM})$ solutions than in no-surfactant samples, there are no doubts that gemini surfactants inhibit AA to a significant extent. This effect obviously makes a great contribution to observed ThT emission signal suppression, even though (i) some visible to naked eye aggregates are deposited in some of the solutions, especially for moderate and longer spacers of gemini surfactant molecule and (ii) that SAXS data reveal protein aggregation for all samples containing gemini surfactants $(20 \mathrm{mM})$. These aggregates may therefore be non-amyloid. The substance which sticks to fibrillar aggregates observed in TEM images may be made of or may contain these non-amyloid aggregates. However, as ThT fluorescence emission signal for oxyC12 (20 $\mathrm{mM}$ ) is very much the same as for other gemini surfactants at the same concentration (Table 3, day 31), despite greater amount of fibrils seen in TEM images, the effect of protein isolation from ThT molecules by gemini surfactants molecules may also be responsible for the ThT signal suppression.

The possible explanation of gemini surfactants inhibitory action may be attributed to their direct interaction with protein misfolded molecules, oligomers, protofibrils and fibrils in a following way. Positively charged gemini surfactant micelles come into interaction with positively charged HEWL aggregates, despite electrostatic repulsion, presumably by hydrophobic interactions. This way micelles cause destabilization of hydrogen bonds responsible for maintaining HEWL fibrillar structure. Because gemini surfactants possess strong inclination of selfaggregation under high surfactant concentrations, they may pull individual HEWL molecules out of fibrillar structure. Hydrophobic chains of surfactant could easily absorb HEWL molecules into gemini micellar structure. ${ }^{24,54}$ Providing HEWL aggregates are exposed to gemini micelles for sufficiently long time, this will eventually lead to a complete fibril disassemble. This mechanism not only would promote aggregates disassemble, but could also efficiently prevent fibrils from their formation. Such mechanism of interaction between a similar gemini surfactant and amyloid beta 1-40 peptide was proposed by Han et al. ${ }^{55}$ As Han's peptide was negatively charged, which significantly supported interaction between surfactant micelles and peptide fibrils, we expect that higher concentration of gemini surfactants are required for effective interaction in a HEWL/ gemini surfactant system, as compared to Han's research. ${ }^{56}$ From this point of view the greater surfactant concentration, the stronger the observed effect, as the efficiency of process of absorbing HEWL molecules into gemini micelles rises with more micelles attached to HEWL aggregates.

Interestingly, gemini surfactants of shorter spacer more effectively inhibited HEWL aggregation. Their spacer is more rigid as compared to the surfactants with longer spacer, due to proximity of positively charged heads linked by the spacer, which tend to be separated from each other. This proximity of two positive charges could make it easier for the surfactant molecule to strongly disturb protein structure, by simultaneous action of the two heads. Nonetheless, the system of surfactant micelles embedding into protein fibrillar structure is quite complex, which makes establishing of exact molecular mechanism difficult.

Sulfobetaines, capable to a very limited action against HEWL amyloidogenesis, have only one hydrophobic chain in each molecule and therefore possess significantly weaker affinity of self-aggregation. ${ }^{55}$ This could presumably be the main reason for their weak anti-amyloid performance.

\subsection{Amyloid aggregation in microfluidic conditions}

We assembled a microfluidic system that would mimic in vivolike conditions of flow in human blood vessels. This system was used in an attempt to model the influence of such a flow on the amyloid formation process. Obviously, we are aware of this model limitations, for instance such as the presence of rigid walls, fixed channel cross section and the flow rate not ideally corresponding to the real in vivo values. However, such a model can test the influence of flow rate alone on amyloidogenesis in channels whose cross-section indeed mimics the diameters of human capillaries.

The bulk measurements reported above were taken as a reference for the microfluidic measurements. A sample containing HEWL under the same conditions as the reference samples (pH, temperature) was placed in the microfluidic chip (described in the Experimental section) and circulated in its channel. At particular time intervals the heating stage was opened to take an aliquot of circulating solution for ex situ measurements.

Fig. 5a shows ThT fluorescence dependence for HEWL sample circulated at an approximate average flow rate of $50 \mu \mathrm{L}$ $\min ^{-1}$. ThT fluorescence curves reveal a very small gradual rise of signal intensity, followed by a sudden dramatic signal increase, which undoubtedly proves that AA took place in the sample, and, reinforcing, to a great extent (greater than in bulk samples), up to about 600 arb. u. However, what is actually an extremely valuable result here, is that amyloid aggregates took not several days, but as little as just several hours to form. This implies an immensely strong effect of circulation on lysozyme 

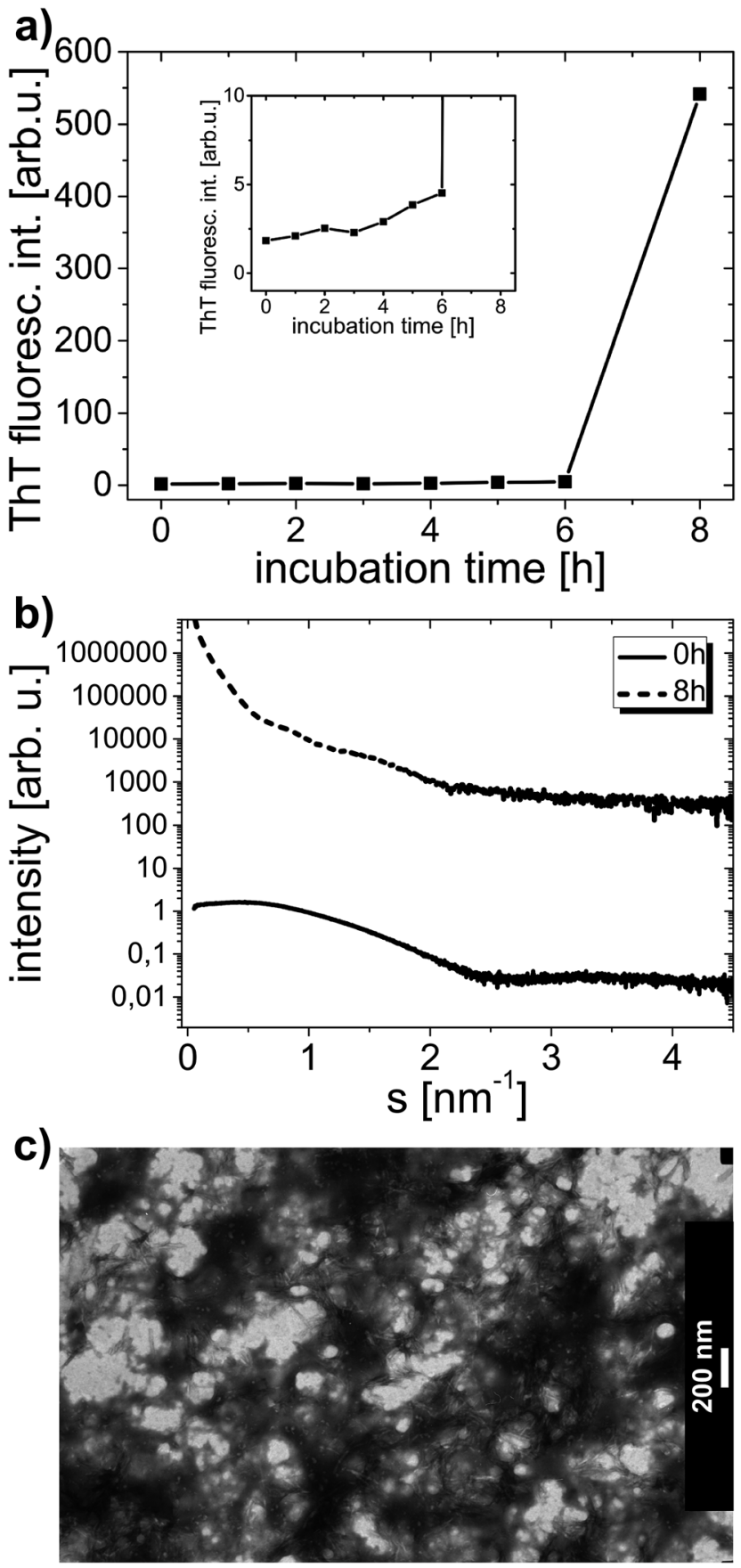

Fig. 5 Thioflavin T fluorescence signal at $\lambda=490 \mathrm{~nm}$ in dependence on time of incubation, collected for the solution of lysozyme without a surfactant during circulation in the microfluidic chip (a). The inset displays the same dependence with a different scale for a better view of data points collected within the first 6 hours. The average flow

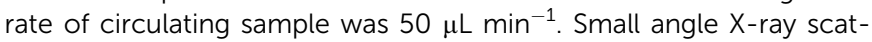
tering curves measured for the sample under study (b). Curves were shifted vertically for clarity. TEM image of the sample after $8 \mathrm{~h}$ of circulation (c).

AA. Fig. 5b shows SAXS patterns with visible characteristic steep slope of the curve at the final stage of the experiment. The curve slope was so steep that no single $R_{\mathrm{g}}$ value could be obtained from this SAXS dependence, which may support the suggestion that the extent of aggregation is greater than in bulk solutions. A dense network of aggregates, comprising plenty of amyloid fibrils formed in the solution after microfluidic flow, can be observed in TEM images. Fig. 5c displays TEM image with very distinct (despite lower visibility of aggregates due to nonuniform stain distribution in sample background) and plentiful short amyloid fibrils shown. Interestingly, the aggregates occurring in this sample seem to have a different morphology than amyloid aggregates formed in bulk conditions. In microfluidic and in bulk conditions alike, some non-negligible distribution of time required for $\mathrm{AA}$ to commence was observed. In two other experiments made, both with the $50 \mu \mathrm{L}$ $\min ^{-1}$ flow rate, AA developed after $3 \mathrm{~h}$ and $5 \mathrm{~h}$ of circulation (there was $200 \mu \mathrm{L}$ of sample at the beginning of both experiments).

This result, that is the finding that much shorter time is required for the amyloid formation in microfluidic conditions, leads to a far-reaching conclusion that the circulation of proteins in human blood vessels could itself have a substantial effect conducive to amyloid aggregates formation. The drastic reduction in the time required for formation of aggregates can presumably be linked to both or one of the following factors: shear forces occurring in the chip and the confinement of the sample. An attempt to study the influence of these factors on aggregation in greater detail could lead to a better understanding of the pathway of, as well as the mechanisms inducing amyloidogenesis. Such considerations are crucial in tackling this fatal disease, as thorough understanding of phenomena governing AA brings seeking for drugs against the disease far more effective.

Lysozyme was also circulated in the microfluidic chip at the approximate flow rates of $100 \mu \mathrm{L} \mathrm{min}^{-1}$ and $20 \mu \mathrm{L} \mathrm{min}{ }^{-1}$. The results obtained for these samples are given in Fig. 6. For the

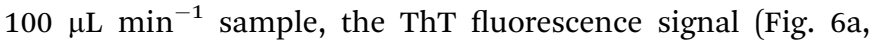
inset) was even higher than for the $50 \mu \mathrm{L} \mathrm{min}{ }^{-1}$ system, reaching almost 900 arb. u. As shown in Fig. 6a, the secondary structure of protein after circulation is strongly modified, with a major minimum in the CD spectrum substantially shifted towards higher wavelength values, with a curve shape similar to that of the $240 \mathrm{~h}$ curve presented in Fig. 3a (when taking into account the long-wavelength range of these two curves). The secondary structure contents were estimated as $39 \% \alpha$-helix and $21 \% \beta$-sheet (as mentioned above, fully conclusive protein concentration values were difficult to be derived). SAXS data, showing the characteristic slope as a result of aggregation, also confirm strong AA (data not shown). For the sample circulated

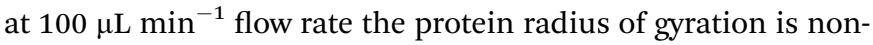
determinable at the end of experiment, just like for the sample circulated at $50 \mu \mathrm{L} \mathrm{m^{-1 }}$ flow rate (Table $\mathrm{S} 2 \dagger$ ). TEM images again reveal a vast number of amyloid fibrils, built in a dense network, in an altered morphology with reference to that in the bulk samples. Fig. 6c displays TEM image of this solution, showing aggregates with morphology very much like the one in the image shown in Fig. 5c. For $20 \mu \mathrm{L} \mathrm{min}^{-1}$ sample, after $9 \mathrm{~h}$ of circulation, a slight growth of ThT fluorescence signal was observed, which alone might be recognized as a presumable indication of AA onset. The $R_{\mathrm{g}}$ calculated for the sample with 20 

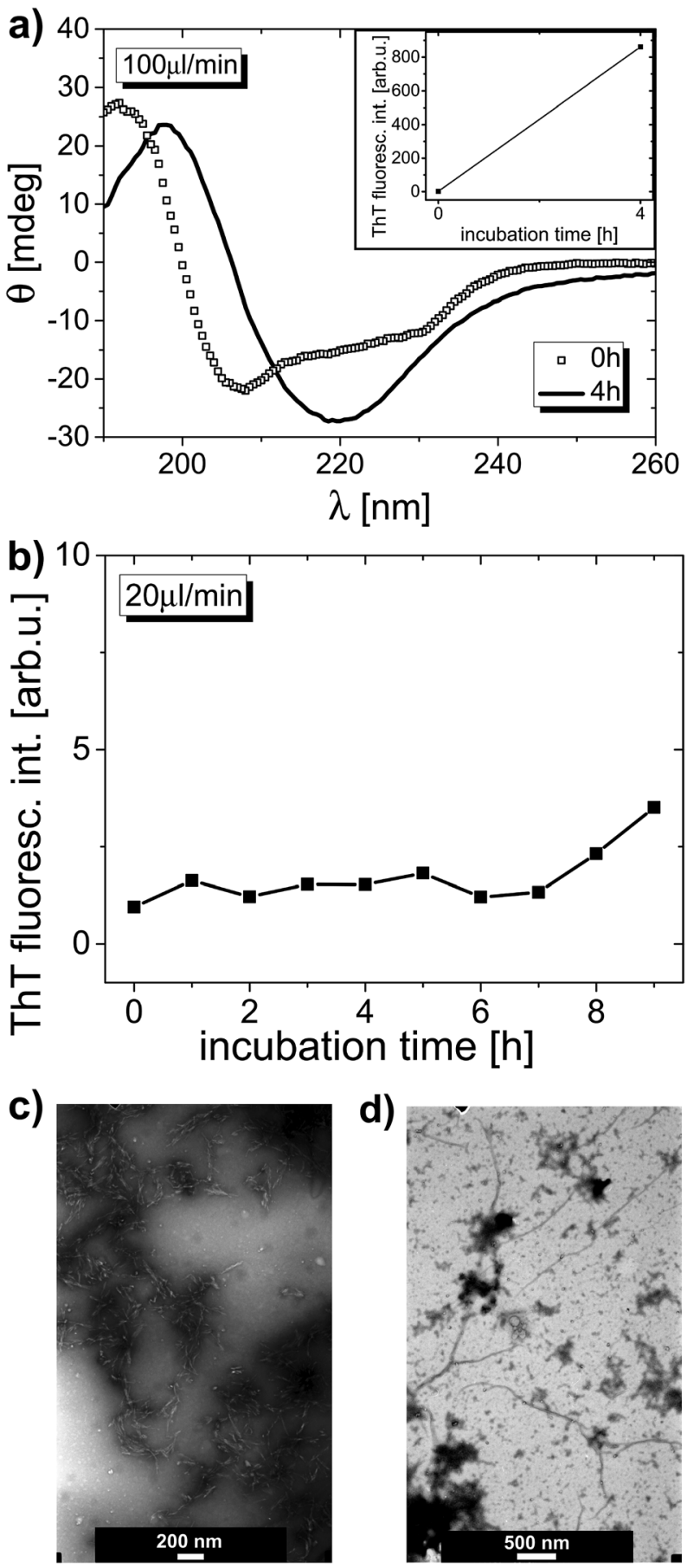

Fig. 6 Circular dichroism curves measured for solution of lysozyme without surfactant after given time of circulation in the microfluidic chip at an average flow rate of $100 \mu \mathrm{L} \mathrm{min}{ }^{-1}$ (a). Thioflavin T fluorescence signal at $\lambda=490 \mathrm{~nm}$ in dependence on time of circulation for the same sample (inset). Thioflavin T fluorescence signal at $\lambda=490 \mathrm{~nm}$ in dependence on time of circulation for solution of lysozyme without surfactant circulating at an average flow rate of $20 \mu \mathrm{L} \mathrm{min}^{-1}$ (b). TEM image of the sample with $100 \mu \mathrm{L} \mathrm{min}{ }^{-1}$ flow rate after $4 \mathrm{~h}$ of circulation (c). TEM image of the sample with $20 \mu \mathrm{L} \mathrm{min}{ }^{-1}$ flow rate after $9 \mathrm{~h}$ of circulation (d).

$\mu \mathrm{L} \min ^{-1}$ flow rate rises insignificantly from $1.5 \mathrm{~nm}$ (prior to incubation) to $1.6 \mathrm{~nm}$ (after $9 \mathrm{~h}$ of circulation). However, TEM images gathered for this sample undoubtedly show that the amyloid aggregation has been started after $9 \mathrm{~h}$ of microfluidic flow. There are distinct long fibrils visible in the images, like the ones shown in the TEM image in Fig. 6d.

Although an attempt to compare the effect of different flow rates on amyloid formation was made, it is still vague which of the flow rates used is the most effective to induce AA. The problem is that such attempt is hampered by a non-negligible distribution of time of AA formation. However, we may expect that the $20 \mu \mathrm{L} \mathrm{min}{ }^{-1}$ sample, due to weaker exposure to shear forces, triggers AA less effectively than the two other flow rates examined. As $20 \mu \mathrm{L} \mathrm{min}^{-1}$-circulating solution encountered smaller shear forces, the AA effect was also smaller, but other experiments with $20 \mu \mathrm{L} \mathrm{min}{ }^{-1}$ flow rate are needed to back this statement.

In addition, we performed two other experiments, testing the possible influence of temperature and $\mathrm{pH}$ on amyloid aggregates formation. Both included one parameter variation, that is: the first experiment - temperature, set at $37{ }^{\circ} \mathrm{C}$ instead of $60^{\circ} \mathrm{C}$, with all other experimental conditions preserved, and the second experiment - $\mathrm{pH}$, set at 7.4 instead of 2.0, with all other parameters, including $60{ }^{\circ} \mathrm{C}$ of incubation temperature, unchanged. Both: increased temperature and decreased $\mathrm{pH}$, with reference to physiological parameters, are essential to induce AA in laboratory conditions in a time scale of a few days. Here we tested whether circulation could be such a strong factor to trigger AA at physiological temperature or physiological $\mathrm{pH}$. The maximum time of circulation in these experiments was increased to 15 hours, because a strong chance of no aggregation to evolve was anticipated. The switch of the flow was additionally computer-assisted and therefore required less experimenter engagement, which helped to enhance the time of measurement. Contrary to this expectation, there actually was observed a minor increase in ThT fluorescence signal after 15 hours of circulation at $37{ }^{\circ} \mathrm{C}$, revealing a supposed onset of AA process (Fig. S4a $\dagger^{\prime}$ ). The ThT signal growth was slight, however distinct. TEM images of this sample give a reliable confirmation of AA occurrence, by catching the moment long fibrils are evidently being formed from smaller subunits (Fig. S4b $\dagger$ ). When circulation was studied at physiological $\mathrm{pH}$, but $60^{\circ} \mathrm{C}$, no sign of amyloid aggregation process was noted (Fig. S4c $\dagger$ ). Also CD results for this sample do not reveal any slightest change in the protein secondary structure (CD data not shown).

Finally, we also made an extra experiment to exclude any contribution to observed effect of fast AA from factors other than the sample circulation. We therefore put HEWL solution into the chip, but without moving it along the chip channel, and applied a typical pressure used in earlier-described microfluidic measurements, of 2500 mbar (although we expected that the pressure used in our experiments is by a few orders of magnitude smaller than that required to induce any AA changes ${ }^{57}$ ). We therefore checked the influence of confinement, pressure applied and wall material (glass, in contrast to polyethylene Eppendorf tubes used for bulk reference solutions). The experiment was stopped after 3 days to avoid chip clogging with protein aggregates. As a result, no ThT fluorescence signal rise for staying-in-the-chip sample was observed (Fig. S4d $\dagger$ ), with a stark conclusion of the crucial role of circulation in inducing 
fast and strong aggregation in the chip, like the AA observed in circulating samples.

Although according to these observations the phenomenon of shear-induced amyloidogenesis is strong and unquestionable, its explanation is not easy to derive. It is a well known circumstance that a protein must overcome a particular potential barrier to misfold and end up in fibrillar aggregates. ${ }^{58}$ According to Dunstan et al., ${ }^{31}$ shear could itself deliver as much as a few $k_{\mathrm{B}} T$ to protein's free energy. Hydrodynamic forces, taking their origin from different velocities which different protein segments have during the laminar flow, could potentially give explanation for protein structure destabilization and unfolding, but, however, claims that the magnitude of hydrodynamic forces could be held responsible for exerting such large effect are raising doubts. ${ }^{59}$ Hill et al. ${ }^{46}$ who examined influence of shear forces on amyloidogenesis of Abeta 1-40 peptide, with use of a different experimental set-up from the microchip used here, reported that the shear forces itself induce formation of amyloid oligomers of this peptide, but not fibrils. The latter are formed as a result of the presence of the oligomers, not directly because of protein exposure to shear force. Beside shear forces, the confinement itself was considered as a factor increasing the aggregation lag time, rather than the opposite. ${ }^{15}$ In microfluidic systems, however, because channel diameter is small, the interface between flowing solution and chip walls is large and confinement is therefore intrinsically responsible for shear forces occurrence during microfluidic flow. In summary, taking all the facts mentioned in this paragraph into account, the research concerning protein solutions and shear forces brings vital findings, but evidently needs further development.

\section{Conclusions}

We have shown that in bulk conditions, at elevated temperature and $\mathrm{pH}$ lower than the physiological value, HEWL protein undergoes evident amyloid aggregation within a few days. The aggregation process is combined with secondary structure modification, including $\beta$-sheet content rise at the expense of $\alpha$ helix content fall. Such results obtained for reference case coincide well with literature data. Addition of dicationic surfactant with a short spacer group between surfactant heads, efficiently hampers this aggregation, but does not totally inhibit AA nor entirely preserve protein secondary structure. Gemini surfactants with longer spacer group, especially oxyC12 surfactant, had also marked inhibitory effect on AA, however noticeably weaker one. Sulfobetaines, on the other hand, had very limited influence on AA, exerting virtually no hampering effect on HEWL amyloidogenesis, although they were able to prevent secondary structure modifications to some extent.

The results obtained from microfluidic experiments show evidently that sample circulation itself has a tremendous impact on amyloid aggregates formation process, as it takes only a few hours for AA to develop in microfluidic flow regime and the extent of amyloid aggregation is even higher than in bulk samples. The secondary structure of HEWL is modified. The structure of aggregates seen in TEM images is different than that in the reference samples. AA is triggered at all three flow rates used. We have also proved that the circulation is such a strong factor conducive to AA formation that it can induce AA in time of several hours also in non-elevated, physiological temperature (with low value of $\mathrm{pH}$ preserved). The fast aggregation effect is probably purely circulation-driven, as the sample placed in the chip without flow, under relevant pressure, does not show the evidence of AA. This result exhibits the role of flow shear forces in the mechanism of amyloid aggregates formation.

\section{Acknowledgements}

The research was supported by a research grant (DEC-2013/09/ N/ST5/02444) from National Science Centre (Poland).

\section{References}

1 A. Aguzzi, Nature, 2014, 512, 32.

2 Y. Avsar, T. Spieker, I. Kabar, C. Roecken, H. Wolters and H. Schmidt, Amyloid, 2014, 21, 128.

3 S. Raccosta, V. Martorana and M. Manno, J. Phys. Chem. B, 2012, 116, 12078.

4 V. M. Trusova and G. P. Gorbenko, J. Photochem. Photobiol., $B, 2012,113,51$.

5 M. R. Mohamadi, Z. Svobodova, R. Verpillot, H. Esselmann, J. Wiltfang, M. Otto, M. Taverna, Z. Bilkova and J.-L. Viovy, Anal. Chem., 2010, 82, 7611.

6 S. Bahramikia, R. Yazdanparast and A. Gheysarzadeh, Chem. Biol. Drug Des., 2012, 80, 227.

7 S. S.-S. Wang, Y.-T. Hung, W.-S. Wen, K.-C. Lin and G.-Y. Chen, Biochim. Biophys. Acta, 2011, 1811, 301.

8 S. Kumar, V. K. Ravi and R. Swaminathan, Biochem. J., 2008, 415, 275.

9 H. Amijee, C. Bate, A. Williams, J. Virdee, R. Jeggo, D. Spanswick, D. I. C. Scopes, J. M. Treherne, S. Mazzitelli, R. Chawner, C. E. Eyers and A. J. Doig, Biochemistry, 2012, 51, 8338.

10 F. Altieri, C. S. Di Stadio, V. Severino, A. Sandomenico, G. Minopoli, G. Miselli, A. Di Maro, M. Ruvo, A. Chambery, V. Quagliariello, M. Masullo, E. Rippa and P. Arcari, Biochimie, 2014, 106, 91.

11 S. Movaghati, A. A. Moosavi-Movahedi, F. Khodagholi, H. Digaleh, E. Kachooei and N. Sheibani, Colloids Surf., B, 2014, $122,341$.

12 I. Choi, Y. S. Huh and D. Erickson, Lab Chip, 2011, 11, 632. 13 J. S. Lee and C. B. Park, Biomaterials, 2010, 31, 6789.

14 R. Kawano, Y. Tsuji, K. Sato, T. Osaki, K. Kamiya, M. Hirano, T. Ide, N. Miki and S. Takeuchi, Sci. Rep., 2013, 3, 1995.

15 T. P. J. Knowles, D. A. White, A. R. Abate, J. J. Agresti, S. I. Cohen, R. A. Sperling, E. J. D. Genst, C. M. Dobson and D. A. Weitz, Proc. Natl. Acad. Sci. U. S. A., 2011, 108, 14746.

16 V. Fodera, S. Pagliara, O. Otto, U. F. Keyser and A. M. Donald, J. Phys. Chem. Lett., 2012, 3, 2803.

17 K. Ziolkowska, R. Kwapiszewski and Z. Brzozka, New J. Chem., 2011, 35, 979. 
18 A. K. Price, D. J. Fischer, R. S. Martin and D. M. Spence, Anal. Chem., 2004, 76, 4849.

19 A. Jain and L. L. Munn, Lab Chip, 2011, 11, 2941.

20 D. Carugo, L. Capretto, S. Willis, A. L. Lewis, D. Grey, M. Hill and X. A. Zhang, Biomed. Microdevices, 2012, 14, 153.

21 X. Ye, L. Lu, M. E. Kolewe, H. Park, B. L. Larson, E. S. Kim and L. E. Freed, Biomaterials, 2013, 34, 10007.

22 I. Pastor, M. L. Ferrer, M. P. Lillo, J. Gomez and C. R. Mateo, J. Phys. Chem. B, 2007, 111, 11603.

23 D. Zhang, H. Li and J.-B. Wang, Int. J. Biol. Macromol., 2015, 72, 243.

24 W. Gospodarczyk, K. Szutkowski and M. Kozak, J. Phys. Chem. B, 2014, 118, 8652.

25 N. Gull, M. A. Mir, J. M. Khan, R. H. Khan, G. M. Rather and A. A. Dar, J. Colloid Interface Sci., 2011, 364, 157.

26 Y. Pi, Y. Shang, C. Peng, H. Liu, Y. Hu and J. Jiang, Biopolymers, 2006, 83, 243.

27 H. Wang, X. Jiang, L. Zhou, Z. Cheng, W. Yin, M. Duan, P. Liu and X. Jiang, J. Lumin., 2013, 134, 138.

28 A. R. Lomasney, L. Yi and M. G. Roper, Anal. Chem., 2013, 85, 7919.

29 T. Mueller, F. S. Ruggeri, A. J. Kulik, U. Shimanovich, T. O. Mason, T. P. J. Knowles and G. Dietler, Lab Chip, 2014, 14, 1315.

30 J. S. Lee, J. Ryu and C. B. Park, Anal. Chem., 2009, 81, 2751.

31 D. E. Dunstan, P. Hamilton-Brown, P. Asimakis, W. Ducker and J. Bertolini, Protein Eng., Des. Sel., 2009, 22, 741.

32 Z. Pietralik, M. Taube, A. Skrzypczak and M. Kozak, Acta Phys. Pol., A, 2010, 117, 311.

33 K. Michocka, D. Wieczorek and R. Zielinski, Polish Patent Appl. P, PL402717-A1, 2013.

34 M. Kozak, K. Szpotkowski, A. Kozak, R. Zielinski, D. Wieczorek and M. J. Gajda, Radiat. Phys. Chem., 2009, 78, S112.

35 M. Kozak, K. Szpotkowski, A. Kozak, R. Zielinski, D. Wieczorek, M. J. Gajda and L. Domka, Radiat. Phys. Chem., 2009, 78, S129.

36 K. Dubey and K. Mar, Biochem. Biophys. Res. Commun., 2014, 448, 480 .

37 L. Whitmore and B. A. Wallace, Nucleic Acids Res., 2004, 32, W668.

38 L. A. Compton and W. C. Johnson, Anal. Biochem., 1986, 155, 155.
39 J. G. Lees, A. J. Miles, F. Wien and B. A. Wallace, Bioinformatics, 2006, 22, 1955.

40 A. R. Round, D. Franke, S. Moritz, R. Huchler, M. Fritsche, D. Malthan, R. Klaering, D. I. Svergun and M. Roessle, J. Appl. Crystallogr., 2008, 41, 913.

41 C. M. Jeffries, M. A. Graewert, D. I. Svergun and C. E. Blanchet, J. Synchrotron Radiat., 2015, 22, 273.

42 C. E. Blanchet, A. Spilotros, F. Schwemmer, M. A. Graewert, A. Kikhney, C. M. Jeffries, D. Franke, D. Mark, R. Zengerle, F. Cipriani, S. Fiedler, M. Roessle and D. I. Svergun, J. Appl. Crystallogr., 2015, 48, 431.

43 T. C. Huang, H. Toraya, T. N. Blanton and Y. Wu, J. Appl. Crystallogr., 1993, 26, 180.

44 P. V. Konarev, V. V. Volkov, A. V. Sokolova, M. H. J. Koch and D. I. Svergun, J. Appl. Crystallogr., 2003, 36, 1277.

45 M. Kozak, J. Appl. Crystallogr., 2005, 38, 555.

46 E. K. Hill, B. Krebs, D. G. Goodall, G. J. Howlett and D. E. Dunstan, Biomacromolecules, 2006, 7, 10.

47 W. Sorteberg, K. F. Lindegaard, K. Rootwelt, A. Dahl, D. Russell, R. Nyberghansen and H. Nornes, Acta Neurochir., 1989, 97, 47.

48 R. M. Lee, Pharmacol. Ther., 1995, 66, 149.

49 S. J. Schreiber, S. Gottschalk, M. Weih, A. Villringer and J. M. Valdueza, Am. J. Neuroradiol., 2000, 21, 1207.

50 M. Qian, L. L. Niu, Y. P. Wang, B. Jiang, Q. F. Jin, C. X. Jiang and H. R. Zheng, Phys. Med. Biol., 2010, 55, 6069.

51 N. Rajaram, A. Gopal, X. J. Zhang and J. W. Tunnell, Lasers Surg. Med., 2010, 42, 680.

52 A. Iram and A. Naeem, Arch. Biochem. Biophys., 2013, 533, 69.

53 I. I. Rzeznicka, R. Pandey, M. Schleeger, M. Bonn and T. Weidner, Langmuir, 2014, 30, 7736.

54 Y. Li, X. Wang and Y. Wang, J. Phys. Chem. B, 2006, 110, 8499. 55 Y. Han, C. He, M. Cao, X. Huang, Y. Wang and Z. Li, Langmuir, 2010, 26, 1583.

56 W. Gospodarczyk and M. Kozak, Colloid Polym. Sci., 2015, 293, 2855.

57 L. Huang, X. Liu, B. Cheng and K. Huang, Arch. Biochem. Biophys., 2015, 568, 46.

58 P. Arosio, T. P. J. Knowles and S. Linse, Phys. Chem. Chem. Phys., 2015, 17, 7606.

59 J. Jaspe and S. J. Hagen, Biophys. J., 2006, 91, 3415. 